MATHEMATICS OF COMPUTATION

Volume 67, Number 224, October 1998, Pages 1617-1635

S $0025-5718(98) 01004-7$

\title{
A SWEEP-PLANE ALGORITHM FOR GENERATING RANDOM TUPLES IN SIMPLE POLYTOPES
}

\author{
JOSEF LEYDOLD AND WOLFGANG HÖRMANN
}

\begin{abstract}
A sweep-plane algorithm of Lawrence for convex polytope computation is adapted to generate random tuples on simple polytopes. In our method an affine hyperplane is swept through the given polytope until a random fraction (sampled from a proper univariate distribution) of the volume of the polytope is covered. Then the intersection of the plane with the polytope is a simple polytope with smaller dimension.

In the second part we apply this method to construct a black-box algorithm for log-concave and $T$-concave multivariate distributions by means of transformed density rejection.
\end{abstract}

\section{INTRODUCTION}

Several methods have been suggested for the generation of uniformly distributed random points on an $n$-polytope $P$. by

(1) If $P$ is a simplex, then by [8, chapter XI.2.5, theorem 2.1] we get such a point

$$
\mathbf{x}=\sum_{j=0}^{n} U_{j} \mathbf{v}_{j} \quad \text { where } \quad \sum_{j=0}^{n} U_{j}=1,
$$

where $\mathbf{v}_{0}, \ldots, \mathbf{v}_{n}$ are the vertices of the simplex $P$ and the $U_{j}$ are generated by a uniform sample on $[0,1]$.

(2) Consequently a method for arbitrary polytopes is to triangulate $P[8$, chapter XI.2.5], i.e. we split the polytopes into $n$-simplices. Triangulation works well for polygons $(\operatorname{dim}(P)=2)$ but is rather difficult for dimension greater than 2 . The complexity of the decomposition step depends on the number of faces, which is $O\left(m^{\lfloor n / 2\rfloor}\right)$, where $m$ is the number of vertices ([23], cf. [8, chapter XI.2.5]).

(3) Another approach is grid methods (see [8, chapter VIII.3.2]). The polytope is enclosed in a hyper-rectangle, which is decomposed into a set of grid rectangles. In a setup step the grid rectangles are classified into inside, outside or on the border of the polytope. Those inside and on the border are stored and randomly chosen by the sampling algorithm. Then a point inside the small rectangle is generated. It is accepted if it is inside the polytope. It is clear that the number of necessary grid rectangles explodes for higher dimensions. Thus for higher dimensions the method has advantages and disadvantages comparable with the next method (4).

Received by the editor February 26, 1997 and, in revised form, August 18, 1997.

1991 Mathematics Subject Classification. Primary 65C10; Secondary 65C05, 68U20.

Key words and phrases. Uniform distributions, polytope, rejection method, multivariate logconcave distributions, universal method. 
(4) A possible variant of (2) and (3) is to enclose $P$ into a simplex (L. Devroye, private correspondence). Here the rejection constant for an arbitrary polytope is of order $O(\operatorname{dim}(P) !)=O(n !)$ but strongly depends on the shape of the polytope. Furthermore it is not a simple task to find the enclosing simplex (e.g. if $P$ has parallel constraints.).

A new approach to the problem is the use of a sweep-plane technique. This technique goes back to Hadwiger [16], [17], who used it in the context of Euler characteristic on the convex ring. It was applied to volume computation by Bieri and Nef [5] and (with a different name) by Lawrence [22] (see also [14]). In [4] a recursive algorithm is used to count the cells of a finite division in $\mathbb{R}^{n}$.

The general idea of sweep-plane algorithms is to "sweep" a hyperplane through a polytope $P$, keeping track of the changes that occur when the hyperplane sweeps through a vertex.

For our purpose the plane is swept through the given simple polytope until a random fraction of the volume of the polytope is covered. This fraction is given by a uniform sample on $[0,1]$. The intersection of the plane with the polytope is a simple polytope with smaller dimension. By recursion we arrive at a polytope of dimension 0 , that is, a single point. The complexity for the first recursion step is $O\left(m^{2}+m n^{3}\right)$.

Although only derived for the convex case, the sweep plane algorithm also works for non-convex polytopes, but in contrast to the methods (2), (3) and (4) only for simple polytopes. Compared with methods (2) and (3), the setup of the new algorithm is much faster but the generation time is slower. Compared to (3) and (4), the new algorithm has the advantage that there is no rejection necessary. The main advantage of our new generation procedure is that the complexity does not grow as fast with the dimension as the methods suggested in literature. There are problems with rounding errors in higher dimensions, but they can be overcome using exact rational arithmetic.

In the second part we apply this method to construct a black-box algorithm for log-concave and $T$-concave multivariate distributions by following the idea of transformed density rejection, introduced in [12] and [18] for the univariate case. The sweep-plane technique is used to generate random variates with respect to the hat function.

\section{SWEEP-PLANE ALGORITHM FOR SIMPLE POLYTOPES}

2.1. Simple convex polytopes. Let us first summarize the concept of polytopes (see [30]; an introduction to convex polytopes can further be found in [15], [3], or from a different point of view in [10], [25]).

An $\mathcal{H}$-polyhedron is an intersection of finitely many closed half-spaces in $\mathbb{R}^{n}$. In linear programming the inequalities $\langle\mathbf{c}, \mathbf{x}\rangle \leq c_{0}$ that define the half-spaces are called constraints. A $\mathcal{V}$-polyhedron is the convex hull of some points in $\mathbb{R}^{n}$. A polyhedron is a point set $P \in \mathbb{R}^{n}$ which can be presented either as a $\mathcal{V}$-polyhedron or as an $\mathcal{H}$-polyhedron. Both representations are equivalent and can be converted to each other (for example, by the reverse search algorithm [2] or the double description method [24], [11]).

A (convex) polytope is a bounded polyhedron. A polyhedron (polytope) of dimension $n$ is called an $n$-polyhedron (n-polytope). A face of a polyhedron is a polyhedron $F \subseteq P$ which is the intersection of $P$ with some supporting hyperplane 
$\left\{\mathbf{x} \in \mathbb{R}^{n}:\langle\mathbf{c}, \mathbf{x}\rangle=c_{0}\right\}$, where $\langle\mathbf{c}, \mathbf{x}\rangle \leq c_{0}$ is satisfied for all points $\mathbf{x} \in P$. A face of dimension $i$ is called an $i$-face. The 0 -faces, 1 -faces and $(n-1)$-faces of an $n$ polytope are respectively its vertices, edges and facets. An $n$-polyhedron is called simple if every vertex is contained in the minimal number of only $n$ facets; that is, only $n$ constraints are binding in each vertex. (In linear programming this is called the non-degenerate case.)

Notice that if we consider any set of constraints that are generic (i.e. they define hyperplanes in general position) then this defines a simple polyhedron. In the generic case an $\mathcal{H}$-polytope is always simple. On the other hand, if we choose points in $\mathbb{R}^{n}$ in general position (i.e. no $n$ of these are affine dependent) then its convex hull is simplicial (all proper faces are simplices) but may not be simple.

\subsection{Sweeping planes.}

Sweep-plane. Let $P$ be a simple convex $n$-polytope and $f(x)$ the density function of the uniform distribution on $P$. For simplicity we set $f(x) \equiv 1$. Choose a nonzero vector $\mathbf{g}$. In what follows we assume

$$
\|\mathbf{g}\|=1 \quad \text { and } \quad\langle\mathbf{g}, \mathbf{x}\rangle \text { is non-constant on every edge in } P
$$

$\langle\cdot, \cdot\rangle$ denotes the scalar product. For a given $\mathbf{x}$ let $x=\langle\mathbf{g}, \mathbf{x}\rangle$. We denote the hyperplane perpendicular to $\mathbf{g}$ through $\mathbf{x}$ by

$$
F(\mathbf{x})=F(x)=\left\{\mathbf{y} \in \mathbb{R}^{n}:\langle\mathbf{g}, \mathbf{y}\rangle=x\right\}
$$

and its intersection with the polytope $P$ by $Q(\mathbf{x})=Q(x)=P \cap F(x) .(F(\mathbf{x})$ and $Q(\mathbf{x})$ depend on $x$ only; thus we write $F(x)$ and $Q(x)$, respectively, if there is no risk of confusion.) $Q(x)$ again is a convex polytope (see [15]). Now we can move this sweep-plane $F(x)$ through the domain $P$ by varying $x$. Figure 1 illustrates the situation. The marginal density function $h_{\mathbf{g}}(x)$ along $\mathbf{g}$ of a uniform distribution with support $P$ is simply given by the volume $A(x)$ of $Q(x)$. We can sample a variate $x$ from the marginal distribution and get the polytope $Q(x)$ (see $\S 2.3$ and $\S 2.4)$.

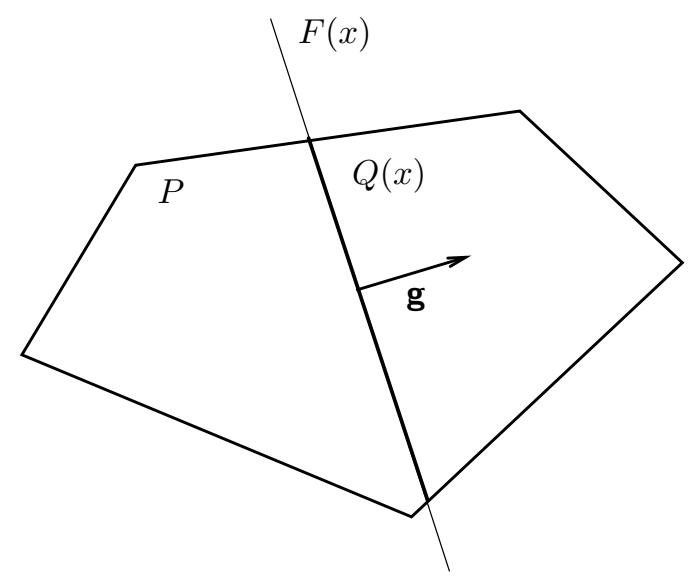

Figure 1. Sweep-plane $F(x)$ 


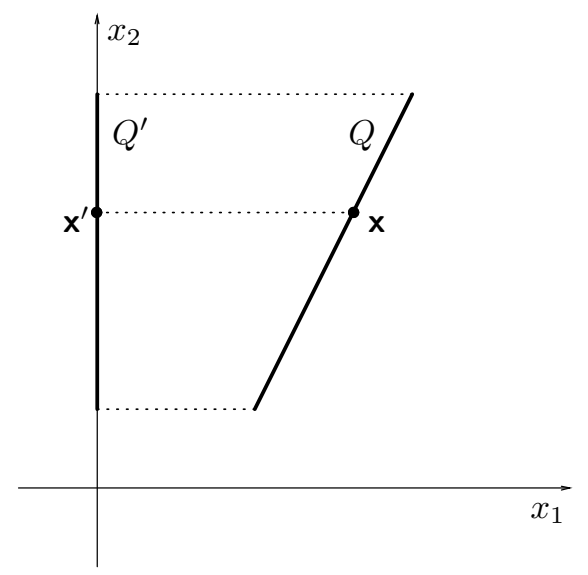

Figure 2. Projection $Q \subset \mathbb{R}^{d} \rightarrow Q^{\prime} \subset \mathbb{R}^{d-1}$

Recursive sweep-plane algorithm. If $\operatorname{dim}(Q(x))=1, Q(x)$ is a line segment and we get the random point by

$$
\mathbf{x}=U \mathbf{v}_{0}+(1-U) \mathbf{v}_{1},
$$

where $\mathbf{v}_{0}, \mathbf{v}_{1}$ are the vertices of $Q(x)$ and $U$ is a uniform sample on $[0,1]$.

If $\operatorname{dim}(Q(x)) \geq 2$ let $Q_{n-1}=Q(x)$. Then $Q_{n-1}$ is a simple $(n-1)$-polytope (immediately from [30, proposition 2.16]). We embed $Q_{n-1}$ into the $\mathbb{R}^{n-1}$ by eliminating the component in $\mathbf{x}$, where $\mathbf{g}$ takes its maximum.

$$
\begin{gathered}
\mathbf{x}=\left(x_{1}, \ldots, x_{M}, \ldots, x_{n}\right) \mapsto \mathbf{x}^{\prime}=\left(x_{1}, \ldots, x_{M-1}, x_{M+1}, \ldots, x_{n}\right) \\
\text { where } \quad g_{M}=\max _{j=1, \ldots, n} g_{j}
\end{gathered}
$$

If the maximum of $g_{j}$ is not unique, we set $M$ equal to the first index that maximizes $g_{j}$.

We get a polytope $Q_{n-1}^{\prime} \subset \mathbb{R}^{n-1}$ (see Figure 2). We choose a proper $\mathbf{g}_{n-1} \in \mathbb{R}^{n-1}$ which satisfies (2), that is, $\left\|\mathbf{g}_{n-1}\right\|=1$ and $\mathbf{x}^{\prime} \mapsto\left\langle\mathbf{g}_{n-1}, \mathbf{x}^{\prime}\right\rangle$ is non-constant on every edge of $Q_{n-1}^{\prime}$. Again we use a sweep plane $F_{n-1}(x)$ and a univariate random number generator to get an $(n-2)$-polytope $Q_{n_{2}}$.

Now we apply the same method to $Q_{n-2}$ and get a polytope $Q_{n-3}$ and so on, until we finish with a polytope $Q_{1}$ of dimension 1 , for which we use (4).

We get the random point $\mathbf{x} \in Q(x)=Q_{n-1}$ from $\mathbf{x}^{\prime} \in Q_{n-2}$ by the fact that $\langle\mathbf{g}, \mathbf{x}\rangle=x$ (see figure 2). Thus we find

$$
\begin{aligned}
& \mathbf{x}=\left(x_{1}, \ldots, x_{n}\right)=\left(y_{1}, \ldots, y_{M-1}, x_{M}, y_{M+1}, \ldots, y_{n}\right) \\
& \text { with } \quad x_{M}=\frac{x-\langle\mathbf{g}, \mathbf{y}\rangle}{g_{M}}, \\
& \text { where } \quad \mathbf{y}=\left(y_{1}, \ldots, y_{n}\right)=\left(x_{1}^{\prime}, \ldots, x_{M-1}^{\prime}, 0, x_{M}^{\prime}, \ldots, x_{n-1}^{\prime}\right) .
\end{aligned}
$$

\subsection{Sample from marginal distribution.}

Computing the volume $A(x)$. Let $F^{-}(x)=\left\{\mathbf{y} \in \mathbb{R}^{n}:\langle\mathbf{g}, \mathbf{y}\rangle \leq x\right\}$. By assumption $P \cap F^{-}(x)$ is bounded. Thus we find that

$$
A(x)=V_{n-1}(P \cap F(x))=\frac{d V_{n}\left(P \cap F^{-}(x)\right)}{d x},
$$




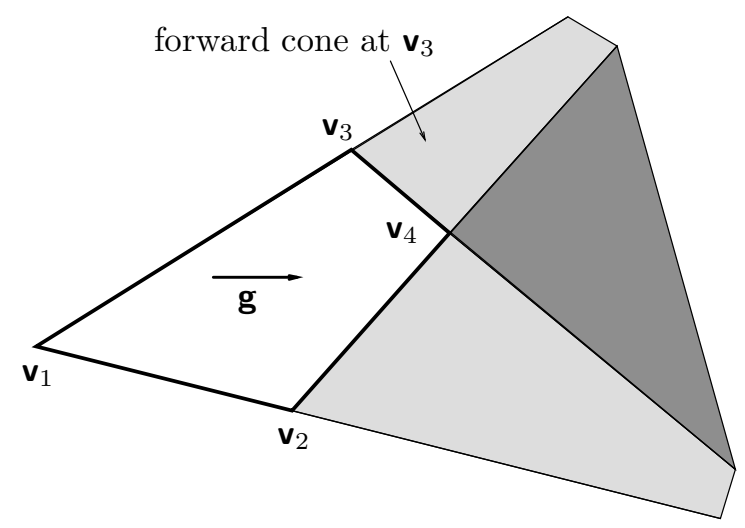

FIgURE 3. Forward cone and Gram's relation

where $V_{k}$ denotes the $k$-dimensional volume. To compute $A(x)$ we modify the method in [5], [22], [14]. Denote the vertices of $P$ by $\mathbf{v}_{j} \in \mathbb{R}^{n}, j=1, \ldots, m$, and $v_{j}=\left\langle\mathbf{g}, \mathbf{v}_{j}\right\rangle$, so that

$$
-\infty<v_{1} \leq v_{2} \leq \ldots \leq v_{m}<\infty .
$$

Additionally we set $v_{0}=-\infty$ and $v_{m+1}=\infty$.

The polytope $P$ can be built up by simple cones at the vertices $\mathbf{v}_{j}$. Let $\mathbf{v}$ be a vertex of $P$ and $\mathbf{t}_{1}^{\mathbf{v}}, \ldots, \mathbf{t}_{n}^{\mathbf{v}}$ be nonzero vectors in the directions of the edges of $P$ (originated from $\mathbf{v}$ ), i.e. for each $j$ and every $\mathbf{x} \in P,\left\langle\mathbf{t}_{j}^{\mathbf{v}}, \mathbf{x}\right\rangle \geq 0$. We define $\gamma_{j}=\operatorname{sgn}\left(\left\langle\mathbf{t}_{j}^{\mathbf{v}}, \mathbf{g}\right\rangle\right)$ and $\delta(\mathbf{v})=\prod_{j=1}^{n} \gamma_{j}$. Notice that by assumption $(2),\left\langle\mathbf{t}_{j}^{\mathrm{v}}, \mathbf{g}\right\rangle \neq 0$. Then the vectors $\gamma_{j} \mathbf{t}_{j}^{\mathbf{v}}$ span the forward cone $C(\mathbf{v})$ at $\mathbf{v}$, i.e.

$$
C(\mathbf{v})=\left\{\mathbf{v}+\sum_{j=1}^{n} c_{j} \gamma_{j} \mathbf{t}_{j}^{\mathbf{v}}: c_{j} \geq 0\right\} .
$$

As can easily be seen, $\langle\mathbf{x}, \mathbf{g}\rangle \geq\langle\mathbf{v}, \mathbf{g}\rangle$ for all $\mathbf{x} \in C(\mathbf{v})$ (see Figure 3 ). Since $P$ is simple, $C(\mathbf{v}) \cap F^{-}(x)$ is an $n$-simplex for $x>v=\langle\mathbf{v}, \mathbf{g}\rangle$, and hence

$$
\begin{aligned}
V_{n}\left(C(\mathbf{v}) \cap F^{-}(x)\right) & =\frac{1}{n !}\left|\operatorname{det}\left((x-v) \frac{\gamma_{1} \mathbf{t}_{1}^{\mathbf{v}}}{\left\langle\gamma_{1} \mathbf{t}_{1}^{\mathbf{v}}, \mathbf{g}\right\rangle}, \ldots,(x-v) \frac{\gamma_{n} \mathbf{t}_{n}^{\mathbf{v}}}{\left\langle\gamma_{N} \mathbf{t}_{n}^{\mathbf{v}}, \mathbf{g}\right\rangle}\right)\right| \\
& =(x-v)^{n} \frac{1}{n !}\left|\operatorname{det}\left(\mathbf{t}_{1}^{\mathbf{v}}, \ldots, \mathbf{t}_{n}^{\mathbf{v}}\right)\right| \delta(\mathbf{v}) \prod_{i=1}^{n}\left\langle\mathbf{t}_{i}^{\mathbf{v}}, \mathbf{g}\right\rangle^{-1}
\end{aligned}
$$

for $x \geq v$, and 0 otherwise.

Let $\chi_{M}$ denote the characteristic function of the set $M \subseteq \mathbb{R}^{n}$, i.e. $\chi_{M}(\mathbf{x})=1$ if $\mathbf{x} \in M$, and 0 otherwise. By a version of Gram's relation (see [27]) it follows ([22]) that

$$
\chi_{P}=\sum_{j=1}^{m} \delta\left(\mathbf{v}_{j}\right) \chi_{C\left(\mathbf{v}_{\mathbf{j}}\right)}
$$

and thus

$$
V_{n}\left(P \cap F^{-}(x)\right)=\sum_{j=1}^{m} \delta\left(\mathbf{v}_{j}\right) V_{n}\left(C\left(\mathbf{v}_{\mathbf{j}}\right) \cap F^{-}(x)\right) .
$$


Define

$$
a_{j}=\frac{1}{(n-1) !}\left|\operatorname{det}\left(\mathbf{t}_{1}^{\mathbf{v}_{j}}, \ldots, \mathbf{t}_{n}^{\mathbf{v}_{j}}\right)\right| \prod_{i=1}^{n}\left\langle\mathbf{t}_{i}^{\mathbf{v}_{j}}, \mathbf{g}\right\rangle^{-1} .
$$

Combining (7), (12), (10), (13) and the fact that $\delta\left(\mathbf{v}_{j}\right)^{2}=1$, we arrive at

$$
A(x)=\sum_{\substack{1 \leq j \leq m \\ v_{j} \leq x}} . a_{j}\left(x-v_{j}\right)^{n-1} .
$$

Using the binomial theorem, we get

$$
A(x)=\sum_{k=0}^{n-1} b_{k}^{(x)} x^{k}
$$

where the coefficients

$$
b_{k}^{(x)}=\left(\begin{array}{c}
n-1 \\
k
\end{array}\right) \sum_{\substack{1 \leq j \leq m \\
v_{j} \leq x}} a_{j}\left(-v_{j}\right)^{n-1-k}
$$

depend on the intervals $\left[v_{j-1}, v_{j}\right)$ only. (Notice that $A(x) \equiv 0$ for $x \geq v_{m}$.)

Marginal density function. The coefficients $b_{k}^{(x)}$ in (15) are constants on the intervals $\left[v_{j-1}, v_{j}\right)$, i.e. $b_{k}^{(x)}=b_{k}^{\left(v_{j-1}\right)}$ for all $x \in\left[v_{j-1}, v_{j}\right)$. On each of these intervals the marginal density function $h_{\mathbf{g}}(x)=A(x)$ is a polynomial of degree $n-1$ with both positive and negative coefficients.

We do not know particular generators for such marginal distributions (except for the special case $x \in\left[v_{1}, v_{2}\right)$, where $A(x)$ is a power function), but we can utilize the fact ([26, theorem 8$])$ that every marginal density of a log-concave distribution again is log-concave (The density of the uniform distribution is constant and thus log-concave.) Since the mode of the distribution is not known and the $b_{k}^{(x)}$ change in every recursion step of the algorithm, it seems most convenient to use the algorithm of [12] on the interval $\left[v_{j-1}, v_{j}\right)$. Other possible choices are the inversion-rejection method (see [8, chapter VII.4]) or (especially in low dimensions) the inversion method.

Marginal distribution function. To find the interval $\left[v_{j-1}, v_{j}\right)$ we need the marginal distribution function $H(x)$; that is,

$$
H(x)=\int_{-\infty}^{x} h_{\mathbf{g}}(t) d t=\int_{-\infty}^{x} \sum_{k=0}^{n-1} b_{k}^{(t)} t^{k} d t .
$$

Generate a uniform sample $U$ on $[0,1]$ and let $H_{P}=H(\infty)$ be the volume of $P$. Then $\mathbf{v}_{j}$ is the least vertex such that

$$
H\left(v_{j-1}\right) \leq U \cdot H_{P}<H\left(v_{j}\right) .
$$

$H(x)$ can be calculated by recursion. Let $v=\max _{\substack{1 \leq j \leq k \\ v_{j} \leq x}} v_{j}$. Then we have

$$
H(x)=H(v)+\sum_{k=0}^{n-1} b_{k}^{(v)} \int_{v}^{x} t^{k} d t
$$


Let

$$
H_{1}=0 \quad \text { and } \quad H_{j}=\sum_{k=0}^{n-1} b_{k}^{\left(v_{j-1}\right)} \int_{v_{j-1}}^{v_{j}} t^{k} d t
$$

the volume of $P \cap\left\{\mathbf{x} \in \mathbb{R}: v_{j-1} \leq x \leq v_{j}\right\}$. Then we find that

$$
H\left(v_{0}\right)=0 \quad \text { and } \quad H\left(v_{j}\right)=H\left(v_{j-1}\right)+H_{j} \quad \text { for } j=1, \ldots, m .
$$

\subsection{Compute $Q(x)$.}

Cut polytopes. We get the vertices of the cut polytope $Q(x)$ by the intersection of the sweep-plane $F(x)$ with all edges $\left(\mathbf{v}_{0}, \mathbf{v}_{1}\right)$ of $P$. Obviously only those edges are of interest where $v_{0} \leq x<v_{1}$. (Again $v_{i}=\left\langle\mathbf{g}, \mathbf{v}_{i}\right\rangle$.) Then we find for the vertex $\mathbf{v}^{\prime}$ of $Q(x)$

$$
\mathbf{v}^{\prime}=\frac{v_{1}-x}{v_{1}-v_{0}} \mathbf{v}_{0}+\frac{x-v_{0}}{v_{1}-v_{0}} \mathbf{v}_{1} .
$$

Hence we get the $\mathcal{V}$-representation of $Q(x)$.

The face lattice. By assumption, a $d$-face of $P$ becomes a $(d-1)$-face of $Q(x)$ when we intersect $P$ with the sweep-plane $F(x)$ (except when $d=0$, i.e. the face is a vertex). To get the cut vertices by (22) we need the incident edges to all vertices of the cut polytopes in all steps of the recursive algorithm. Thus we need the face lattice (also called incident graph in [10]), i.e. the set of faces partially ordered by inclusion (see [30, chapter 2.2]). For determination, notice that every point $\mathbf{x} \in P$ is an element of one or more facets. Thus we introduce an index $\rho(\mathbf{x})$ (see [2], [5]). Let $f_{1}, \ldots, f_{N}$ be the facets of $P$ (in arbitrary but fixed order). Then we set

$$
\rho(\mathbf{x})=\left(\rho_{1}(\mathbf{x}), \ldots, \rho_{N}(\mathbf{x})\right) \quad \text { with } \rho_{i}(\mathbf{x})= \begin{cases}1 & \text { if } \mathbf{x} \in f_{i}, \\ 0 & \text { otherwise }\end{cases}
$$

Let

and

$$
\rho(\mathbf{x}) \leq \rho(\mathbf{y}) \quad \Leftrightarrow \quad \rho_{i}(\mathbf{x}) \leq \rho_{i}(\mathbf{y}) \quad \forall i=1, \ldots, N
$$

Notice that $\rho(\mathbf{x})=\rho(\mathbf{y})$ if and only if $\mathbf{x}$ and $\mathbf{y}$ are in the relative interior of the same face $f$. Thus we get an index for each face by

$$
\rho(f)=\rho(\mathbf{x}) \text { for an } \mathbf{x} \in \text { relative interior of } f .
$$

By means of this index we can find the face lattice. Since $P$ is simple by assumption, we have for faces $f, f_{1}$ and $f_{2}$

$$
\begin{gathered}
f_{1} \supseteq f_{2} \quad \Leftrightarrow \quad \rho\left(f_{1}\right) \leq \rho\left(f_{2}\right), \\
\operatorname{dim}(f)=n-|\rho(f)| .
\end{gathered}
$$

Two $d$-faces $f_{1}$ and $f_{2}$ are joined by a $(d+1)$-face if and only if

$$
\rho\left(f_{1}\right)>\rho(f) \text { and } \rho\left(f_{2}\right)>\rho(f) .
$$

Using (28), this is equivalent to

$$
\left|\rho\left(f_{1}\right) \wedge \rho\left(f_{2}\right)\right|=\left|\rho\left(f_{1}\right)\right|-1,
$$

where $\wedge$ denotes the bitwise AND-operator. 
Recursion step. The $d$-faces of $P$ become the $(d-1)$-faces of $Q(x)$. We use the same indices for the faces of the cut polytopes $Q$. Problems arise if $\left\langle\mathbf{g}, \mathbf{v}_{0}\right\rangle=x$ in (22), i.e. the sweep-plane $F(x)$ contains a vertex $\mathbf{v}_{0}$ of $P$. Then there might be two or more edges with the same vertex $\mathbf{v}_{0}$. We get $\mathbf{v}_{0}$ as a vertex of $Q(x)$ at least twice, but with different indices since the indices of the edges differ. In this case we append $\mathbf{v}_{0}$ to the vertex list of $Q(x)$ (without changing its index) and ignore the incident edges.

Let $Q_{k}$ be the cut polytope after $n-k$ steps. Thus it has dimension $k$. Because of assumption (2), (28) holds analogously, except when $f$ is a vertex:

$$
\operatorname{dim}(f)=k-|\rho(f)| \quad \text { if } f \text { is not a vertex of } Q_{k} .
$$

Moreover, (29) holds. But (30) is valid only if the faces $f_{i}$ are not vertices of the cut polytope.

2.5. The algorithm. We are given the $\mathcal{H}$ - or $\mathcal{V}$-representation of a simple convex $n$-polytope. (Notice that the convex hull of $n+2$ or more points in general position in $\mathbb{R}^{n}$ is not a simple polytope. An $\mathcal{H}$-polyhedron might not be bounded, and thus the uniform distribution over the polyhedron does not exist. In both cases the algorithm does not work.) The algorithm $\operatorname{sweEP}()$ requires a list of the vertices $\mathbf{v}_{j}$ of $P$ (the $\mathcal{V}$-representation) and all the indices $\rho\left(\mathbf{v}_{j}\right)$ of these vertices. E.g. [1], [6] or [11] do the job. Some of these programs offer options to check the required conditions. Then algorithm SweEP() runs as follows:

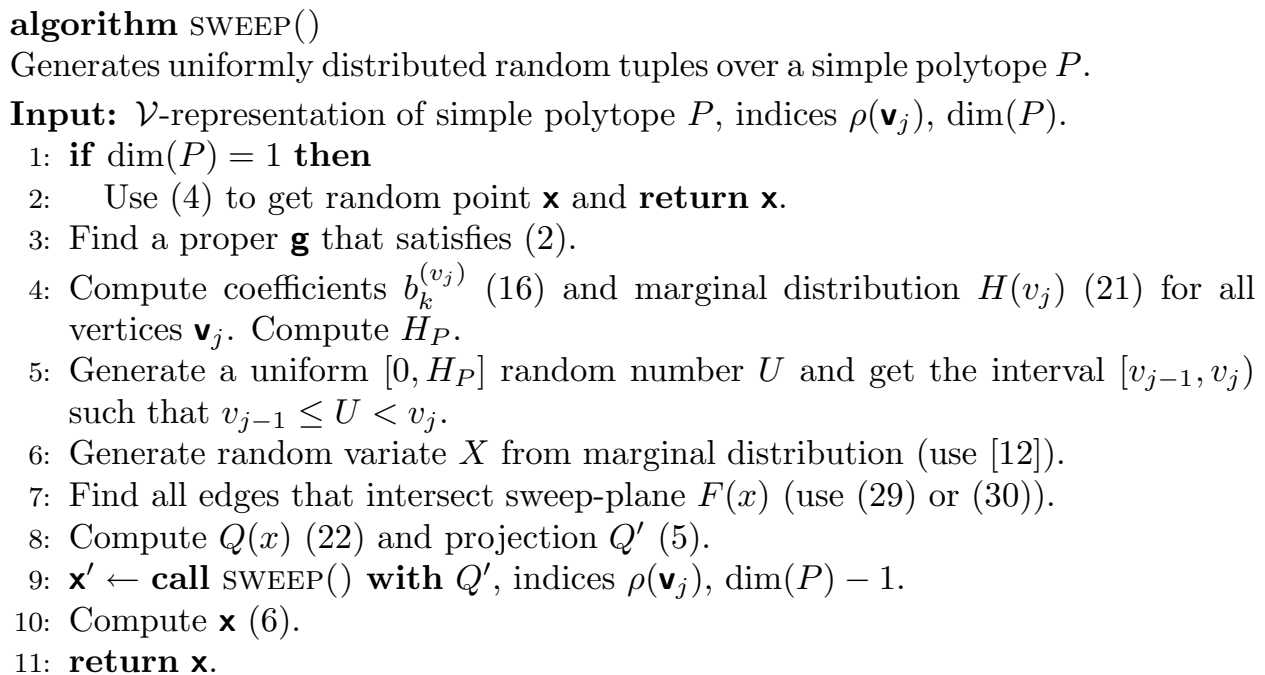

\subsection{Remarks.}

The choice of $\mathbf{g}$. The algorithm is sensible about the choice of $\mathbf{g}$. The method requires summing a lot of numbers for computing the coefficients $b_{k}^{\left(v_{j}\right)}$ in (16). Some of these numbers are positive, some negative. If $\langle\mathbf{g}, \mathbf{x}\rangle$ is "nearly" constant on an edge of $P$, then these numbers can be quite large in magnitude, so that there can be considerable loss of significance due to round-off errors. As a consequence $A\left(v_{m}\right) \neq 0$ at the last vertex of the polytope. This is likely to happen for an arbitrary $\mathbf{g}$ if dimension is high and $P$ has many faces (and vertices). 
In the literature this problem is not really solved. [22] gives a solution only for the case when rational arithmetic is used for computation. [5] suggests the use of randomly chosen vectors.

A possible solution to this problem is: (1) Choose a g. (2) Calculate all coefficients $a_{j}$. (3) If one of these is too big (e.g. larger than $10^{5}$ ) reject $\mathbf{g}$ and try another one. A good choice for $\mathbf{g}$ are the unit vectors $(0, \ldots, 1, \ldots, 0)$ (which are easy to use). If these do not work, possible choices are random vectors (as suggested in [5]) or the vectors $\mathbf{v}_{j}-\overline{\mathbf{v}}$, where $\overline{\mathbf{v}}=\frac{1}{m} \sum_{i=1}^{m} \mathbf{v}_{i}$ denotes the barycenter of $P$. Another possibility is to apply small random rotations of the axis and try the unit vectors again.

Non-convex polytopes. This sweep-plane algorithm can be modified for non-convex simple polytopes. The polytope $P$ can be presented in Boolean form, the face lattice (again) by the indices $\rho(f)$ (see [5] for details).

Complexity. We assume that the $\mathcal{V}$-representation $P$ is given. For computing the marginal density $h_{\mathbf{g}}(x)=A(x)$ we need determinants at all vertices of $P$, a task taking $O\left(m n^{3}\right)$ steps. To get all vertices of the cut polytope $Q(x)$ in $\S 2.4$ we have to find all edges of $P$ that intersect the sweep plane, which is of order $O\left(m^{2}\right)$. Thus for the first recursion step the amount of work is $O\left(m^{2}+m n^{3}\right)$.

Comparison. It is difficult to compare the sweep-plane algorithm with the rejection methods (3) and (4) of the introduction. There the rejection constants for an arbitrary polytope grow with $O(n$ !). On the other hand, there are of course the special cases of the hyperrectangle and the simplex, respectively, where these methods are optimal. In contrast to method (3), no algorithm to construct an enclosing simplex is available in the literature for method (4).

Compared with the triangluation method (2), the main advantage of the new algorithm is the fact that practically no setup is necessary. On the other hand, sampling is slower. For method (2) the complexity of the decomposition step depends on the number of faces, which is $O\left(m^{\lfloor n / 2\rfloor}\right)$, where $m$ is the number of vertices ([23]). Thus for polytopes with a large number of vertices in high dimensions triangulation is very slow. Hence our new method is preferable if

- the dimension is high $(\geq 3)$, and

- the polytope contains a large number of vertices, or

- we only need a few random points for the given polytope.

Volume computation. The generation of random tuples in a polytope is closely related to the determination of volumes. Volume computation is reported to be P-hard (cf. [9], [14]).

\section{A REJECTION TEChNique FOR MULTIVARIATE LOG-CONCAVE DENSITIES}

For the generation of variates from bivariate and multivariate distributions papers are rare. Only the generation of the multinormal and of the Wishart distribution are well known and discussed (see e.g. [8] and [7]). One approach - especially considered by researchers interested in simulation - aims to develop new, easy to generate classes of multivariate distributions; it is only necessary (and possible) to specify the marginal distribution and the degree of dependence measured by some correlation coefficient (see the monograph [21]). This idea seems to be attractive for most simulation practitioners interested in multivariate distributions, but it is no help if we want to generate variates from a distribution with given density. 
The conditional distribution method requires the knowledge of and the ability to sample from the marginal and the conditional distributions (see [8, chapter XI.1.2]).

The multivariate extension of the ratio of uniforms method as described in [28] and [29] can be reformulated as rejection from a small family of table-mountain shaped multivariate distributions. This point of view explains the poor acceptance probability for high correlation. The practical problem of how to obtain the enclosing multivariate rectangle for the ratio of uniforms method is not discussed in these papers and seems to be difficult for most distributions.

To our knowledge, no universal algorithms are known for multivariate distributions with given density function. In [8, chapter XI.1.3] it is even stressed that no general inequalities for multivariate densities are available, a fact which makes it impossible to design black-box algorithms similar to those in [8] for the univariate case. In [19] a universal algorithm for log-concave distributions is developed for the bivariate case. It uses the idea of transformed density rejection, which is presented in a first form in [8, chapter VII.2.4] and with a different set-up in [12].

In this section we generalize this idea to the multivariate case. The sweep-plane technique is used to sample from the hat function.

\subsection{Transformed density rejection.}

Density. We are given a multivariate distribution with differentiable density function

$$
f: D \rightarrow[0, \infty), \quad D \subseteq \mathbb{R}^{n}, \quad \text { with mode } \mathbf{m} .
$$

For simplicity we assume $D=\mathbb{R}^{n}$.

Transformation. To design a black-box algorithm utilizing the rejection method it is necessary to find an automatic way to construct a hat function for a given density. Transformed density rejection, introduced under a different name in [12] and generalized in [18], is based on the idea that the density $f$ is transformed by a monotone $T$ (e.g. $T(x)=\log (x))$ in such a way that (see [18]):

(T1) $\tilde{f}(\mathbf{x})=T(f(\mathbf{x}))$ is concave. We then say " $f$ is $T$-concave".

(T2) $\lim _{x \rightarrow 0} T(x)=-\infty$;

(T3) $T(x)$ is differentiable and $T^{\prime}(x)>0$, which implies $T^{-1}$ exists; and

(T4) the volume under the hat is finite.

Hat. It is then easy to construct a hat $\tilde{h}(\mathbf{x})$ for $\tilde{f}(\mathbf{x})$ as the minimum of $N$ tangents. Since $\tilde{f}(\mathbf{x})$ is concave, we clearly have $\tilde{f}(\mathbf{x}) \leq \tilde{h}(\mathbf{x})$ for all $\mathbf{x} \in \mathbb{R}^{n}$. Transforming $\tilde{h}(\mathbf{x})$ back into the original scale, we get $h(\mathbf{x})=T^{-1}(\tilde{h}(\mathbf{x}))$ as majorizing function or hat for $f$, i.e. with $f(\mathbf{x}) \leq h(\mathbf{x})$. Figure 4 illustrates the situation for the univariate case by means of the normal distribution and the transformation $T(x)=\log (x)$. The left hand side shows the transformed density with three tangents. The right hand side shows the density function with the resulting hat. Rejection. The basic form of the multivariate rejection method is given by

\section{algorithm REJECTION()}

1: Set-up: Construct a hat function $h(\mathbf{x})$.

2: Generate a random tuple $\mathbf{X}=\left(X_{1}, \ldots, X_{n}\right)$ with density proportional to $h(\mathbf{X})$ and a uniform random number $U$.

3: If $U h(\mathbf{X}) \leq f(\mathbf{X})$ return $\mathbf{X}$ else go to 2 .

The main idea of this section is to extend transformed density rejection to the multivariate case. 

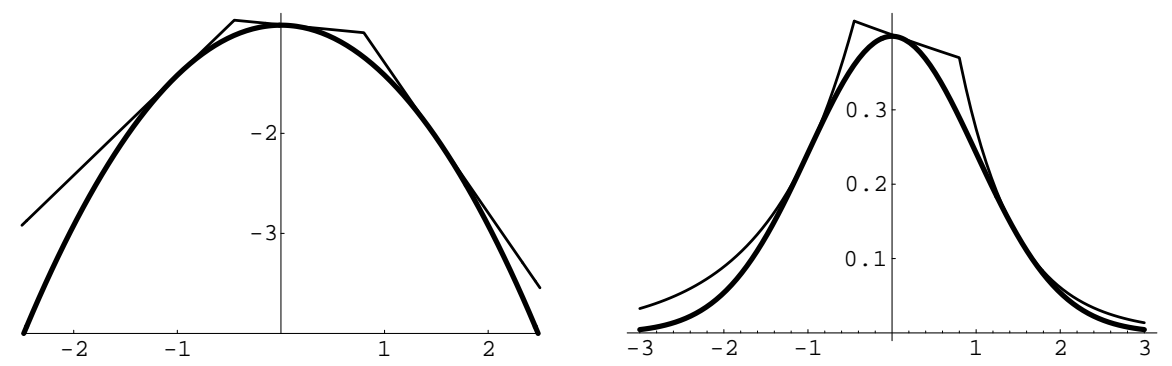

FIgURE 4. Hat function for univariate normal density

\subsection{Construct a hat function.}

Tangents. To construct the hat function we choose $N$ points $\mathbf{p}_{i} \in D \subseteq \mathbb{R}^{n}$ and take tangents $\ell_{i}(\mathbf{x})$ of the transformed density $\tilde{f}$ at these points:

$$
\ell_{i}(\mathbf{x})=\tilde{f}\left(\mathbf{p}_{i}\right)+\left\langle\nabla \tilde{f}\left(\mathbf{p}_{i}\right),\left(\mathbf{x}-\mathbf{p}_{i}\right)\right\rangle .
$$

The hat is then the pointwise minimum of these tangents:

$$
\tilde{h}(\mathbf{x})=\min _{i=1, \ldots, m} \ell_{i}(\mathbf{x}) \quad \text { and } \quad h(\mathbf{x})=\exp (\tilde{h}(\mathbf{x})) .
$$

Although the main idea of multivariate transformed density rejection is simple, it is still hard work to collect all the necessary details. One problem is the suitable choice of the points $\mathbf{p}_{i}$. In the univariate case we are able to optimize the choice of the points (see [18] and [20]). It is even possible to show that the execution time of the algorithm is uniformly bounded for a family of $T$-concave distributions. In the multivariate case this task seems to be impracticable. So we use the important idea of adaptive rejection sampling introduced in [12].

Adaptive rejection sampling. Adapted to our situation, it works in the following way: For the start take at least $n+1$ points of contact, which only must have the property that the volume below the hat $h(\mathbf{x})$ is bounded. Then start the generation of random variates with this hat until a point $\mathbf{x}$ is rejected. Now use $\mathbf{x}$ to construct an additional tangent and thus a new hat, and restart generation of random points. Every rejected point is taken for an additional tangent until the maximum number $N$ of tangents is reached. The points of contact are thus chosen by a stochastic algorithm, and it is clear that the multivariate density of the distribution of the next point for a new tangent is proportional to $h(\mathbf{x})-f(\mathbf{x})$. Hence with $N$ tending towards infinity the acceptance probability for a hat constructed in such a way converges to 1 with probability 1 .

It is not difficult to show that the expected rejection constant is of order $1+$ $O\left(N^{-2 / n}\right)$ : Since $h$ is constructed by tangents over each polytope, we find that $h(\mathbf{x})-f(\mathbf{x})=O\left(\left\|\mathbf{x}-\mathbf{p}_{i}\right\|^{2}\right)$ for the polytope $P_{i}$, and thus the volume between $h$ and $f$ in a ball is of order $O\left(\left\|\mathbf{x}-\mathbf{p}_{i}\right\|^{2+n}\right)$. Without loss, $D$ is bounded. The volume of each polytope is $O\left(d^{n}\right)$, where $d$ is the diameter of $P_{i}$. Assuming that all polytopes have the same volume, we find $d=O\left(N^{-1 / n}\right)$. Thus the total volume between $h$ and $f$ is given by $N \cdot O\left(\left(N^{-1 / n}\right)^{2+n}\right)=O\left(N^{-2 / n}\right)$. Since the polytopes do not have 
the same size but are constructed in order to minimize this volume, the statement follows.

3.3. Generate random tuples. We use a modified version of the sweep-plane algorithm in $\S 2$ to generate random tuples with respect to the hat function.

Polyhedra. The domain in which a particular tangent $\ell_{i}$ determines the hat function $h$ is a convex $n$-polyhedron which may be bounded or not. We find, for a touching point $\mathbf{p}_{i}$ and its tangent $\ell_{i}(\mathbf{x})$,

$$
P_{i}=\left\{\mathbf{x} \in \mathbb{R}^{n}: h(\mathbf{x})=T^{-1}\left(\ell_{i}(\mathbf{x})\right)\right\}=\bigcap_{j=1, \ldots, N}\left\{\mathbf{x} \in \mathbb{R}^{n}: \ell_{i}(\mathbf{x}) \leq \ell_{j}(\mathbf{x})\right\} .
$$

To avoid lots of indices we write $\mathbf{p}, \ell(\mathbf{x})$ and $P$ without the index $i$ if there is no risk of confusion.

We make the following assumptions about the polyhedron $P$ :

(P1) $P$ is a simple polyhedron.

(P2) There exists a maximum of $\ell$ in $P$.

(P3) $\ell$ is non-constant on every edge of $P$.

We always can find touching points such that these restrictions hold.

Sweep-plane algorithm. In each of these polyhedra, $\ell(\mathbf{x})$ is constant on every intersection of $P$ with an affine hyperspace (called a flat) perpendicular to the gradient of $\ell(\mathbf{x})$. Since by condition $(\mathrm{P} 3) \nabla \ell=\nabla \tilde{f}(\mathbf{p}) \neq 0$, let

$$
\mathbf{g}=-\frac{\nabla \tilde{f}(\mathbf{p})}{\|\nabla \tilde{f}(\mathbf{p})\|}
$$

Let again $x=\langle\mathbf{g}, \mathbf{x}\rangle . F(x)$ denotes the sweep-plane and $Q(x)$ its intersection with $P$ (see $\S 2.2)$. Notice that under conditions (P1)-(P3) all the sweep-plane technique derived in $\S 2$ still works for an unbounded polyhedron $P$. By conditions (P2) and (P3) the cut polytope $Q(x)$ is bounded. By setting

$$
\alpha=\tilde{f}(\mathbf{p})-\langle\nabla \tilde{f}(\mathbf{p}), \mathbf{p}\rangle \quad \text { and } \quad \beta=\|\nabla \tilde{f}(\mathbf{p})\|
$$

and by inserting into (33) we find for the hat function in $P$

$$
\left.h\right|_{P}(\mathbf{x})=T^{-1}(\ell(\mathbf{x}))=T^{-1}(\alpha-\beta x) .
$$

The marginal density function $h_{\mathbf{g}}$ of the hat $\left.h\right|_{P}$ along $\mathbf{g}$ is then

$$
h_{\mathbf{g}}(x)=\int_{Q(x)} h(\mathbf{y}) d F(x)=A(x) \cdot T^{-1}(\alpha-\beta x),
$$

where the volume $A(x)$ of $Q(x)$ is given by (15).

Log-concave densities. The transformation $T(x)=\log (x)$ satisfies (T1)-(T4). If $T(f(\mathbf{x}))=\log (f(\mathbf{x}))$ is concave, we say $f$ is log-concave.

We have $T^{-1}(x)=\exp (x)$, and thus by (39) and (15)

$$
h_{\mathbf{g}}(x)=\exp (\alpha-\beta x) \sum_{k=0}^{n-1} b_{k}^{(x)} x^{k} .
$$

$h_{\mathbf{g}}$ is again log-concave by [26, theorem 8$]$, since it is the marginal density of the $\log$-concave function $\left.\exp (\ell(\mathbf{x}))\right|_{P}$. Again it is best to use the algorithm of [12] on the intervals $\left[v_{j-1}, v_{j}\right)($ see $\S 2.3)$. 
The marginal distribution function $H(x)=\int_{-\infty}^{x} h_{\mathbf{g}}(t) d t$ is calculated analogously to (19) and (21) by recursion. For volume $H_{j}$ below the hat in the interval $\left[v_{j-1}, v_{j}\right)$ we now have

$$
H_{j}=e^{\alpha} \sum_{k=0}^{n-1} b_{k}^{\left(v_{j-1}\right)} \int_{v_{j-1}}^{v_{j}}(\beta t)^{k} e^{\beta t} d t .
$$

By substituting $z=\beta t$ and using formula (2.323) in [13] we arrive at

$$
\begin{aligned}
H_{j}= & e^{\alpha} \sum_{k=0}^{n-1} b_{k}^{\left(v_{j-1}\right)} \beta^{-k-1} k ! \\
& \cdot\left(e^{-\beta v_{j-1}} \sum_{l=0}^{k} \frac{\left(\beta v_{j-1}\right)^{l}}{l !}-e^{-\beta v_{j}} \sum_{l=0}^{k} \frac{\left(\beta v_{j}\right)^{l}}{l !}\right),
\end{aligned}
$$

and for the unbounded interval $\left[v_{m}, \infty\right)$ (if $P$ is not bounded)

$$
H_{m+1}=e^{\alpha} \sum_{k=0}^{n-1} b_{k}^{\left(v_{m}\right)} k ! \beta^{-k-1} e^{-\beta v_{m}} \sum_{l=0}^{k} \frac{\left(\beta v_{m}\right)^{l}}{l !} .
$$

Sample from marginal distribution. For the algorithm of [12] we first have to take one of the $N$ polyhedra $P_{i}$ and one of the intervals $\left[v_{j-1}, v_{j}\right)$ on this polyhedron. The latter is done by (18).

Let $H_{\text {tot }}=\sum_{P_{i}} H_{P_{i}}$ denote the total volume below the hat and $U$ a random point from a uniform sample on $[0,1]$. Then we select the polytope $P_{j}$ such that

$$
\sum_{i=1}^{j-1} H_{P_{i}} \leq U \cdot H_{t o t}<\sum_{i=1}^{j} H_{P_{i}} .
$$

3.4. Construct polyhedra. There are two differences from $\S 2$. (1) Some of the polyhedra are not bounded. (2) We cannot use e.g. [11] to compute all the vertices of the polyhedra whenever we add a new construction point.

Vertices and extreme rays. For an unbounded polyhedron we have vertices and extreme rays. The latter may be interpreted as "vertices at infinity". The set of all vertices of the polyhedron (in $\mathbb{R}^{n}$ or at infinity) can be written as $\mathbb{R}^{n} \cup S^{n-1}$, where $S^{n-1}$ denotes the unit sphere in $\mathbb{R}^{n}$. For an algebraic description of this set, we define a space $\mathbb{R}_{\infty}^{n}$ by

$$
\begin{aligned}
& \mathbb{R}_{\infty}^{n}=\left\{\left(x_{0} ; \mathbf{x}\right): x_{0} \in \mathbb{R}, \mathbf{x} \in \mathbb{R}^{n},\left(x_{0} ; \mathbf{x}\right) \neq(0 ; \mathbf{0}), x_{0} \geq 0\right\}, \\
& \mathbf{x}=\mathbf{y} \quad \Leftrightarrow \quad x_{i}=\alpha y_{i} \text { for an } \alpha>0 \text { for all } i=0, \ldots, n .
\end{aligned}
$$

As can easily be seen, we have $\left\{\left(x_{0} ; \mathbf{x}\right) \in \mathbb{R}_{\infty}^{n}: x_{0} \neq 0\right\} \cong \mathbb{R}^{n}$ and $\left\{\left(x_{0} ; \mathbf{x}\right) \in\right.$ $\left.\mathbb{R}_{\infty}^{n}: x_{0}=0\right\} \cong S^{n-1}$. We may write $\mathbb{R}_{\infty}^{n} \cong \mathbb{R}^{n} \cup S^{n-1}$. Notice that we get the projective space $\mathbb{P R}^{n}$ by identifying antipodal points in the subset $S^{n-1}$. In other words, we use the projective space $\mathbb{P R}^{n}$ but distinguish between two directions of points at infinity. For that reason we call $P_{0}=\left\{\left(x_{0} ; \mathbf{x}\right) \in \mathbb{R}_{\infty}^{n}: x_{0}=0\right\}$ the hyperplane at infinity (although it is not "really" a hyperplane). The facets of $P_{i}$ in $\mathbb{R}_{\infty}^{n}$ are given by the equalities $\ell_{i}\left(\frac{\mathbf{x}}{x_{0}}\right)=\ell_{j}\left(\frac{\mathbf{x}}{x_{0}}\right)$. We use the standard Euclidean metric in the subsets $\left\{\left(x_{0} ; \mathbf{x}\right) \in \mathbb{R}_{\infty}^{n}: x_{0} \neq 0\right\}$ and $\left\{\left(x_{0} ; \mathbf{x}\right) \in \mathbb{R}_{\infty}^{n}: x_{0}=0\right\}$ (this cannot be extended to the whole space). 
We embed the polyhedron $P$ into the compact $\mathbb{R}_{\infty}^{n}$ set by

$$
\begin{aligned}
& \mathbf{v} \in \mathbb{R}^{n} \hookrightarrow(1 ; \mathbf{v})=\left(1 ; v_{1}, \ldots, v_{n}\right) \in\left\{\left(x_{0} ; \mathbf{x}\right) \in \mathbb{R}_{\infty}^{n}: x_{0} \neq 0\right\} \\
& \quad \text { for a vertex } \mathbf{v} \text { of } P, \\
& \mathbf{t} \in \mathbb{R}^{n} \hookrightarrow(0 ; \mathbf{t})=\left(0 ; t_{1}, \ldots, t_{n}\right) \in\left\{\left(x_{0} ; \mathbf{x}\right) \in \mathbb{R}_{\infty}^{n}: x_{0}=0\right\}
\end{aligned}
$$

for a nonzero vector $\mathbf{t}$ in the directions of edges of $P$.

An unbounded convex polyhedron is then a polyhedron with vertices in the hyperplane at infinity. The coordinates of a vertex at infinity are given by the vector in the direction of its corresponding unbounded edge, i.e. the extreme ray.

We call all faces with vertices in $\mathbb{R}^{n}$ and in the hyperplane at infinity unbounded faces, and the faces with all vertices at hyperplane at infinity faces at infinity.

For a point $\mathbf{x}$ at infinity we set $\ell(\mathbf{x})=\langle\nabla \ell, \mathbf{x}\rangle$, i.e. $\|\mathbf{x}\|$ times the directional derivative of $\ell$ along $\mathbf{x}$.

Edges. To calculate the coefficients $a_{j}$ in (13) we need the vectors $\mathbf{t}_{i}^{\mathbf{v}}$ in the directions of the edges of $P$. Using the embedding into $\mathbb{R}_{\infty}^{n}$, we find for an edge $\left(\mathbf{v}_{0}, \mathbf{v}_{1}\right)$, $\mathbf{v}_{0} \in \mathbb{R}^{n}$, that

$$
(0 ; \mathbf{t})= \begin{cases}\left(1 ; \mathbf{v}_{1}\right)-\left(1 ; \mathbf{v}_{0}\right) & \text { if the edge is bounded } \\ \left(0 ; \mathbf{v}_{1}\right) & \text { if the edge is unbounded }\end{cases}
$$

The points on such an edge are then given by

$$
\mathbf{v}=\left\{\begin{array}{lll}
(1-t) \mathbf{v}_{0}+t \mathbf{v}_{1}, & 0 \leq t \leq 1, & \text { if edge }\left(\mathbf{v}_{0}, \mathbf{v}_{1}\right) \text { is bounded } \\
\mathbf{v}_{0}+t \mathbf{v}_{1}, & t \geq 0, & \text { if } \mathbf{v}_{0} \in \mathbb{R}^{n} \text { and } \mathbf{v}_{1} \text { is at infinity } \\
t_{0} \mathbf{v}_{0}+t_{1} \mathbf{v}_{1}, & t_{0}, t_{1}>0, & \text { if edge }\left(\mathbf{v}_{0}, \mathbf{v}_{1}\right) \text { is at infinity }
\end{array}\right.
$$

In the last case $t_{0} \mathbf{v}_{0}+t_{1} \mathbf{v}_{1}$ again is a point at infinity, and thus any multiple of this vector gives the same point.

Index $\rho(\mathbf{x})$ and face lattice. The indices $\rho(\mathbf{x})$ and $\rho(f)$ are defined similarly to $\S 2.4$. Now we use the polyhedra $P_{i}$ rather than facets:

$$
\begin{gathered}
\rho(\mathbf{x})=\left(\rho_{0}(\mathbf{x}) ; \rho_{1}(\mathbf{x}), \ldots, \rho_{N}(\mathbf{x})\right), \\
\text { with } \quad \rho_{i}(\mathbf{x})= \begin{cases}1 & \text { if } \mathbf{x} \in P_{i}, \\
0 & \text { otherwise. }\end{cases}
\end{gathered}
$$

$P_{0}$ denotes the hyperplane at infinity. Every vertex $\mathbf{v}$ is an element of at least $n+1$ polyhedra. From now on we assume that

$\left(\mathrm{P}^{\prime}\right)$ every (finite) vertex $\mathbf{v}$ is an element of exactly $n+1$ polyhedra.

Notice that each vertex is an element of at least $n+1$ polyhedra. Since we choose the construction points at random, this assumption holds with probability 1 if the Hessian of the transformed density is non-degenerate almost everywhere.

Using $\left(\mathrm{P}^{\prime}\right)$, the definitions and equations of $\S 2.4$ are still valid; except (28), which now is given by

$$
\operatorname{dim}(f)=n+1-|\rho(f)| .
$$

Initial polyhedra. We start the algorithm with $n+1$ points for constructing the hat function. We can choose the vertices of a regular $n$-simplex, centered at the mode m. For example, such points are given by

$$
\begin{aligned}
& \mathbf{p}_{j}=\sigma \sqrt{\frac{n+1}{n}}\left((a, a, a, \ldots, a)+\mathbf{e}_{j}+\mathbf{m}\right), \quad j=1, \ldots, n, \\
& \mathbf{p}_{n+1}=\sigma \sqrt{\frac{n+1}{n}}((b, b, b, \ldots, b)+\mathbf{m}) .
\end{aligned}
$$


Here $\mathbf{e}_{j}$ denotes the $j$-th unit-vector and $b=-\frac{1}{\sqrt{n+1}}, a=-\frac{b+1}{n}$. The parameter $\sigma$ can be chosen appropriately for the distribution.

We have one vertex in $\mathbb{R}^{n}$ and $n+1$ vertices at infinity for the initial polyhedra. For all these vertices $\mathbf{v}_{j}$ we find $\rho_{j}(\mathbf{v})=0$ for exactly one $j$. We get the coordinates of the vertex $\mathbf{v}_{j}$ by solving the following system of equalities in $\mathbb{R}_{\infty}^{n}$ :

$$
\begin{aligned}
& \rho_{0}\left(\mathbf{v}_{j}\right) \cdot x_{0}=0, \\
& \rho_{j}\left(\mathbf{v}_{j}\right) \cdot\left(\ell_{n+1}(\mathbf{x})-\ell_{j}(\mathbf{x})\right)=0 \quad \text { for } j=1, \ldots, n .
\end{aligned}
$$

Notice that one of these equalities vanishes, since $\rho_{j}\left(\mathbf{v}_{j}\right)=0$. To get the coordinates of the vertices at infinity we have to determine the direction of each solution $\left(0 ; \mathbf{t}_{i}\right)$. We must have

$$
\ell_{i}\left(\mathbf{v}+\mathbf{t}_{i}\right)>\ell_{j}\left(\mathbf{v}+\mathbf{t}_{i}\right) \quad \text { for at least one } j \neq i .
$$

By means of the index $\rho(\mathbf{v})$ we can easily verify which vertices belong to which initial polytopes $P_{i}$.

Violated conditions. If condition $(\mathrm{P} 1),\left(\mathrm{P} 1^{\prime}\right)$ or $(\mathrm{P} 3)$ fails, we have to move the vertices of the regular simplex in (50) a little bit by adding a random point.

Condition (P2) might fail, if the gradient of the transformed density $\nabla \tilde{f}(\mathbf{x})$ is "far away" from pointing to the mode. For example, this happens for the normal density $\left.f(x, y)=\exp \left(-x^{2}-1000 y^{2}\right)\right)$. A possible solution to this problem is to start with the points $\pm \mathbf{e}_{j}$ as construction points. (But then we have more than $n+1$ vertices for the $2 n$ initial polyhedra.) Because of the convexity of the transformed density, condition (P2) always holds if each polyhedron can be moved into the cone $\left\{\mathbf{x} \in \mathbb{R}^{n}: x_{i} \geq 0\right\}$.

Adding a construction point. For the adaptive method it is necessary to add a new construction point whenever a point is rejected. Notice that $\ell(\mathbf{x})$ gives the directional derivative for points at infinity with $\|\mathbf{x}\|=1$. Then the following procedure gives the new polyhedra:

(A1) get the new tangent $\ell(\mathbf{x})$ at this point (see (33));

(A2) find all edges $\left(\mathbf{v}_{0}, \mathbf{v}_{1}\right)$ with $\ell\left(\mathbf{v}_{0}\right)<\tilde{h}\left(\mathbf{v}_{0}\right)$ and $\ell\left(\mathbf{v}_{1}\right)>\tilde{h}\left(\mathbf{v}_{1}\right)$;

(A3) calculate the new vertex $\mathbf{v}=t_{0} \mathbf{v}_{0}+t_{1} \mathbf{v}_{1}$, such that $\ell(\mathbf{v})=\tilde{h}(\mathbf{v})$. Using (47) and the linearity of $\ell$ and $\tilde{h}$ on every edge, we find that

\begin{tabular}{ccc|cc} 
case & $\mathbf{v}_{0}$ & $\mathbf{v}_{1}$ & $t_{0}$ & $t_{1}$ \\
$\in \mathbb{R}^{n}$ & $\in \mathbb{R}^{n}$ & $\frac{\delta_{1}}{\delta_{1}-\delta_{0}}$ & $\frac{\delta_{0}}{\delta_{0}-\delta_{1}}$ \\
$\in \mathbb{R}^{n}$ & inf & 1 & $-\frac{\delta_{0}}{\delta_{1}}$ \\
inf & $\in \mathbb{R}^{n}$ & $-\frac{\delta_{1}}{\delta_{0}}$ & 1 \\
inf & inf & $\delta_{1}$ & $\delta_{0}$
\end{tabular}

where

$$
\delta_{i}=\ell\left(\mathbf{v}_{i}\right)-\tilde{h}\left(\mathbf{v}_{i}\right)
$$

(A4) remove all vertices $\mathbf{v}$ (and adjacent faces) with $\ell(\mathbf{v})<\tilde{h}(\mathbf{v})$;

(A5) check assumptions (P1)-(P3):

$(\mathrm{P} 1)$ and $\left(\mathrm{P}^{\prime}\right)$ are violated if we find a new vertex $\mathbf{v}$ with $\ell(\mathbf{v})=\tilde{h}(\mathbf{v})$.

(P3) fails if we find two new vertices $\mathbf{v}_{0}$ and $\mathbf{v}_{1}$, adjacent by an edge, with $\ell\left(\mathbf{v}_{0}\right)=\ell\left(\mathbf{v}_{1}\right)$.

(P2) always holds, if it holds for the initial polytope. 
If one of the assumptions (P1)-(P3) is violated, we cannot use the point as a new construction point.

\subsection{Remarks.}

A C-implementation. We translated our algorithm into a working C-program. We tested the algorithm for the multivariate normal distribution. The program worked well up to dimensions $6-7$. Then round-off errors occur too frequently, i.e. some of the rejected points could not be used for constructing the hat and many trials have to be made to find a vector $\mathbf{g}$ for the sweep-planes in the cut polytopes $Q_{j}$. Furthermore the average number of generated random points per second decreases rapidly, which is due to the great number of vertices of the polytopes $P_{i}$.

\subsection{Possible variants.}

Subset of $\mathbb{R}^{n}$ as domain. Obviously we can restrict the domain $D$ of density $f$ to a simple polyhedron, which may be bounded or not. Changes to the basic version are: (1) The initial polyhedra have more vertices (and thus the usage of a vertex finding algorithm, e.g. [2], is recommended). (2) The index $\rho(\mathbf{x})$ must be extended by the facets of the boundary of $D$, i.e we append $\rho_{-i}(\mathbf{x})=1$ if $\mathbf{x}$ is in the boundary facet $f_{i}$, and $\rho_{-i}(\mathbf{x})=0$ otherwise.

The mode as touching point. When we use the mode $\mathbf{m}$ of the density $f$ as touching point for our hat, $\ell(\mathbf{x})$ is constant on the corresponding polytope $P_{\mathbf{m}}$, since $\nabla \tilde{f}(\mathbf{m})=$ 0 . Therefore we can use the algorithm from $\S 2$. (But we have to check if this polyhedron is bounded.)

Problems arise for all neighboring polyhedra $P_{i}$. Each of these must have an edge common with $P_{\mathbf{m}} \cdot \ell_{i}(\mathbf{x})$ is constant on this edge and hence assumption (P3) fails for all $P_{i}$. This assumption is necessary for calculating $A(x)$ in (14). Thus we "ignore" the mode $\mathbf{m}$ when we construct the other polyhedra (see Figure 5) and modify the marginal density in (39) to

$$
\begin{gathered}
h_{\mathbf{g}}(x)= \begin{cases}A(x) \cdot T^{-1}(\alpha-\beta x) & \text { for } x \geq v_{l}, \\
0 & \text { for } x<v_{l},\end{cases} \\
\text { where } \quad v_{l}=\min _{\substack{\mathbf{x} \in P \\
\ell(\mathbf{x})=\tilde{h}(\mathbf{x})}}\langle\mathbf{g}, \mathbf{x}\rangle .
\end{gathered}
$$

If $P$ does not intersect with the polytope $P_{\mathbf{m}}$, then $v_{l}=v_{1}$.

$T_{c}$-concave densities. A family $T_{c}$ of transformations that fulfill conditions (T1)(T4) is introduced in [18]. Let $c \leq 0$. Then we set

\begin{tabular}{c|ll|ll}
$c$ & & $T_{c}(x)$ & $T_{c}^{-1}(x)$ & \multicolumn{1}{c}{$T_{c}^{\prime}(x)$} \\
\hline$c=0$ & $\mathbb{R}^{+} \rightarrow \mathbb{R}$ & $\log (x)$ & $\exp (x)$ & $x^{-1}$ \\
$-\frac{1}{n}<c \leq 0$ & $\mathbb{R}^{+} \rightarrow \mathbb{R}^{-}$ & $-x^{c}$ & $(-x)^{1 / c}$ & $-c x^{c-1}$
\end{tabular}

It can easily be verified that condition (T4) holds if and only if $-\frac{1}{n}<c \leq 0$. Moreover for $c<0$ we must have $\left.h\right|_{P}<0$. To ensure the negativity of the hat we always have to choose the mode $\mathbf{m}$ as construction point for a tangent plane $\ell$.

In [18] it was shown that if a density $f$ is $T_{c}$-concave, then it is $T_{c_{1}}$-concave for all $c_{1} \leq c$. 


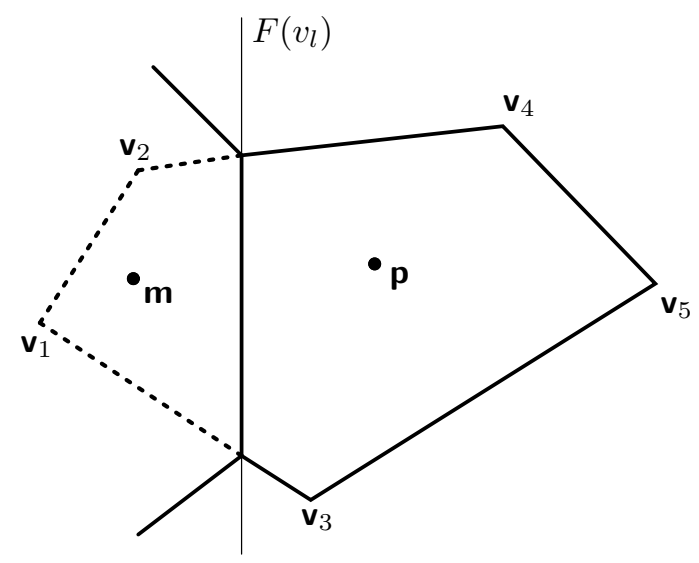

FiguRE 5. "Hidden" part of a polytope

The case $c=0$ is already described in $\S 3.3$. For the case $c<0$ the marginal density function is given by

$$
h_{\mathbf{g}}(x)=(\beta x-\alpha)^{\frac{1}{c}} \sum_{k=0}^{n-1} b_{k}^{(x)} x^{k} .
$$

Notice that $\alpha-\beta t<0$ for all $t \geq v_{1}$, and that $\frac{1}{c}<-n$. By substituting $z=\beta t-\alpha$ and using the binomial theorem we find, for the marginal distribution function,

$$
\begin{aligned}
H_{j}= & \sum_{k=0}^{n-1} b_{k}^{\left(v_{j-1}\right)} \beta^{-k-1} \sum_{l=0}^{k}\left(\begin{array}{l}
k \\
l
\end{array}\right) \frac{1}{l+\frac{1}{c}+1} \alpha^{k-l} \\
& \cdot\left(\left(\beta v_{j}-\alpha\right)^{l+\frac{1}{c}+1}-\left(\beta v_{j-1}-\alpha\right)^{l+\frac{1}{c}+1}\right),
\end{aligned}
$$

and for the unbounded interval $\left[v_{m}, \infty\right)$ (if $P$ is not bounded)

$$
H_{m+1}=-\sum_{k=0}^{n-1} b_{k}^{\left(v_{m}\right)} \beta^{-k-1} \sum_{l=0}^{k}\left(\begin{array}{l}
k \\
l
\end{array}\right) \frac{1}{l+\frac{1}{c}+1} \alpha^{k-l}\left(\beta v_{m}-\alpha\right)^{l+\frac{1}{c}+1} .
$$

Due to the following lemma we can use the algorithm of [18] to generate random points in the intervals $\left[v_{j-1}, v_{j}\right)$ with respect to this marginal distribution.

Lemma 1. The marginal density $h_{\mathbf{g}}(x)=(\beta x-\alpha)^{\frac{1}{c}} A(x)\left(-\frac{1}{n}<c \leq 0\right)$ is $T_{c^{-}}$ concave.

Proof. It remains to prove that $T_{c}\left(h_{\mathbf{g}}(x)\right)=(\alpha-\beta x) A(x)^{c}$ is concave for $c<0$. Let $g(x)=\alpha-\beta x$ and $f(x)=A(x)^{c}$. Let $x_{2}>x_{1} \geq v_{1}$ and $\bar{x}=h x_{1}+(1-h) x_{2}$. By assumption, $g\left(x_{2}\right) \leq g(\bar{x}) \leq g\left(x_{1}\right)<0$ and $g(\bar{x})=h g\left(x_{1}\right)+(1-h) g\left(x_{2}\right)$. $f(x)$ is convex, since $A(x)$ is log-concave (see $\S 2.3$ ) and hence $T_{c}(A(x))=-A(x)^{c}$ is concave. We have to show that

$$
\begin{aligned}
f(\bar{x}) & g(\bar{x})-\left(h f\left(x_{1}\right) g\left(x_{1}\right)+(1-h) f\left(x_{2}\right) g\left(x_{2}\right)\right) \\
& =h g\left(x_{1}\right)\left(f(\bar{x})-f\left(x_{1}\right)\right)+(1-h)\left(f(\bar{x})-f\left(x_{2}\right)\right) \geq 0 .
\end{aligned}
$$

Notice that $f(\bar{x}) \leq h f\left(x_{1}\right)+(1-h) f\left(x_{2}\right)$ by assumption. Thus the left hand side of $(58)$ is $\geq h(1-h)\left(g\left(x_{1}\right)-g\left(x_{2}\right)\right)\left(f\left(x_{2}\right)-f\left(x_{1}\right)\right)$, which is $\geq 0$ if $f\left(x_{1}\right) \leq f\left(x_{2}\right)$. 
For the case $f\left(x_{1}\right) \geq f\left(x_{2}\right)$ notice that, by the convexity of $f(x), f\left(x_{1}\right)-f(\bar{x}) \geq$ $(1-h)\left(f\left(x_{1}\right)-f\left(x_{2}\right)\right)$ and $f(\bar{x})-f\left(x_{2}\right) \leq h\left(f\left(x_{1}\right)-f\left(x_{2}\right)\right)$. Thus the left hand side of $(58)$ is $\geq h(1-h)\left(g\left(x_{1}\right)-g\left(x_{2}\right)\right)\left(f\left(x_{1}\right)-f\left(x_{2}\right)\right) \geq 0$, as proposed.

\section{ACKNOWLEDGEMENTS}

We wish to note our appreciation for help rendered by Jörg Lenneis. He has given

us lots of hints for the implementation of the algorithm. We also thank Gerhard Derflinger for his interest in our work.

\section{REFERENCES}

1. D. Avis, A C implementation of the reverse search vertex enumeration algorithm, Tech. report, School of Computer Science, McGill University, Montreal, Quebec, 1993, report and code available at ftp://mutt.cs.mcgill.ca/pub/C.

2. D. Avis and K. Fukuda, A pivoting algorithm for convex hull and vertex enumeration of arrangements and polyhedra, Discrete Comput. Geom. 8 (1992), 295-313. MR 93h:68137

3. M. Berger, Geometrie, 2 ed., vol. 3, Convexes et polytopes, polyedres reguliers, aires et volumes. Fernand Nathan, Paris, 1978. MR 81k:51001c

4. H. Bieri and W. Nef, A recursive sweep-plane algorithm, determining all cells of a finite division of $R^{m}$, Computing 28 (1982), 189-198. MR 83k:52014

5. _ A sweep-plane algorithm for the volume of polyhedra represented in boolean form, Linear Algebra Appl. 52/53 (1983), 69-97. MR 85h:52007

6. Th. Christof, Porta - a polyhedron representation transformation, Universität Heidelberg, code available at ftp://elib.zib-berlin.de/pub/mathprog.

7. J. Dagpunar, Principles of random variate generation, Clarendon Press, Oxford, 1988. MR 90d: 65020

8. L. Devroye, Nonuniform random variate generation, Springer-Verlag, New-York, 1986. MR 87i:65012

9. M. E. Dyer and A. M. Frieze, On the complexity of computing the volume of a polyhedron, SIAM J. Comput. 17 (1988), no. 5, 967-974. MR 90f:68077

10. H. Edelsbrunner, Algorithms in combinatorial geometry, EATCS Monographs on Theoretical Computer Science, vol. 10, Springer-Verlag, 1987. MR 89a:68025

11. K. Fukuda, cdd reference manual, Tech. report, ETH Zentrum, Zürich, Switzerland, 1995, report and code availeable at ftp://ifor13.ethz.ch/pub/fukuda/cdd.

12. W. R. Gilks and P. Wild, Adaptive rejection sampling for Gibbs sampling, Appl. Statistics 41 (1992), 337-348

13. I. S. Gradshteyn and I. M. Ryzhnik, Table of integrals, series, and products, 5th ed., Academic Press, 1965. MR 33:5952

14. P. Gritzmann and V. Klee, On the complexity of some basic problems in computational convexity. II: Volume and mixed volumes. Polytopes: abstract, convex and computational, (Scarborough, Ontario, Canada) (T. Bisztriczky et al., eds.), NATO ASI Series, Ser. C, Math. Phys. Sci., no. 440, NATO Advanced Study Institute, Kluwer Academic Publishers, 1994, pp. 373-466. MR 96k:52012

15. B. Grünbaum, Convex polytopes, Interscience, 1967. MR 37:2085

16. H. Hadwiger, Eulers Charakteristik und kombinatorische Geometrie, J. Reine Angew. Math. 194 (1955), 101-110. MR 17:402a

17. _ Eine Schnittrekursion für die Eulersche Charakteristik euklidischer Polyeder, Elem. Math. 23 (1968), no. 6, 121-132. MR 38:5112

18. W. Hörmann, A rejection technique for sampling from T-concave distributions, ACM Trans. Math. Software 21 (1995), no. 2, 182-193. MR 96b:65018

19., A universal generator for bivariate log-concave distributions, Computing 52 (1994), 89-96. MR 94j:65014

20. W. Hörmann and G. Derflinger, Universal generators for correlation induction, Compstat, Proceedings in Computational Statistics (Heidelberg) (R. Dutter and W. Grossmann, eds.), Physica-Verlag, 1994, pp. 52-57. MR 95j:62003

21. M. E. Johnson, Multivariate statistical simulation, John Wiley \& Sons, New York, 1987. 
22. J. Lawrence, Polytope volume computation, Math. Comput. 57 (1991), no. 195, 259-271. MR 91j:52019

23. P. McMullen, The maximum number of faces of a convex polytope, Mathematika 17 (1970), 179-184. MR 44:921

24. T. S. Motzkin, H. Raiffa, G. L. Thompson, and R. M. Thrall, The double description method, Contribution to the Theory of Games, Vol. II (H. W. Kuhn and A. W. Tucker, eds.), Annals of Math. Studies, vol. 28, Princton University Press, 1953, pp. 81-103. MR 15:638g

25. W. Nef, Beiträge zur Theorie der Polyeder, Herbert Lang, Bern, 1978. MR 58:18152

26. A. Prékopa, On logarithmic concave measures and functions, Acta Sci. Math. Hungarica 34 (1973), 335-343. MR 53:8357

27. G. C. Shephard, An elementary proof of Gram's theorem for convex polytopes, Canad. J. Math. 19 (1967), 1214-1217. MR 37:822

28. S. Stefanescu and I. Vaduva, On computer generation of random vectors by transformations of uniformly distributed vectors, Computing 39 (1987), 141-153. MR 89i:65008

29. J. C. Wakefield, A. E. Gelfand, and A. F. M. Smith, Efficient generation of random variates via the ratio-of-uniforms method, Statist. Comput. 1 (1991), 129-133.

30. G. M. Ziegler, Lectures on polytopes, Graduate Texts in Mathematics, vol. 152, SpringerVerlag, New York, 1995. MR 96a:52011

University of Economics and Business Administration, Department for Applied Statistics and Data Processing, Augasse 2-6, A-1090 Vienna, Austria

E-mail address: Josef.Leydold@wu-wien.ac.at

University of Economics and Business Administration, Department for Applied Statistics and Data Processing, Augasse 2-6, A-1090 Vienna, Austria

Current address: Boğaziçi University, Department of Industrial Engineering, 80815 BebekIstanbul, Turkey

E-mail address: whoer@statrix2.wu-wien.ac.at 